\title{
Simple and Cost Effective System for Overall Equipment Efficiency Measurement
}

\author{
T. Rautio ${ }^{1}$, K. Kutuniva ${ }^{2}$, J. Mäkikangas ${ }^{3}$, K. Mäntyjärvi ${ }^{4}$ \\ Kerttu Saalasti Institute, University of Oulu, Nivala, FINLAND \\ ${ }^{1}$ timo.rautiodoulu.fi \\ ${ }^{2}$ kari.kutunivadoulu.fi \\ ${ }^{3}$ jarmo.makikangas@oulu.fi \\ ${ }^{4}$ kari.mantyjarvi@oulu.fi
}

\begin{abstract}
Today's business environment and intense competition drives organizations to find improved and more efficient ways to develop their production. For many manufacturers one of the key challenges is to find the bottle necks of the current production line. Overall Equipment Efficiency measurement can be used to find these, but the available solutions are usually expensive and specialized equipment. The purpose of this paper is to demonstrate how hobbyist level electronics and very cost-effective solutions can successfully be used to measure Overall Equipment Efficiency. Such a system was developed and tested in a factory environment.
\end{abstract}

\section{INTRODUCTION}

The Overall Equipment Efficiency (OEE) has gained wide and growing recognition over the past few decades as a score for production line efficiency. It was first introduced by Nakajima [1] as a metric for Total Productive Maintenance (TPM) that was suggested to maximize equipment effectiveness by people-intensive, preventive maintenance system involving the whole organization. OEE value can also be calculated for one production line or machine and therefore the performance comparison between the production lines and machines is possible [2]. In the most simplified way, a crude estimation for the OEE can be presented as

$$
O E E=\frac{\text { valuable operating time }}{\text { loading time }}
$$

In other words, OEE represents the time production line was doing its job as a percentage of the time the production should have been running. While simple, this alone gives very little information about the underlying reasons for the OEE variation. Slicing the OEE into smaller components describing the different kinds of losses in production gives more detailed data that can be used to improve the production efficiency. Nakajima separated six big losses in the production process which are categorized in three groups presented in Table I.

Eliminating or at least reducing all of these losses results in improved overall efficiency. More precise value for the OEE can be calculated from the three components as follows:

$$
O E E=\text { Availability } * \text { Performance } * \text { Quality }
$$

TABLE I

6 BIG LOSSES [1]

\begin{tabular}{c|c}
\hline Efficiency component & 6 Big losses \\
\hline \hline Availability & $\begin{array}{c}\text { Breakdowns } \\
\text { Setups/Adjustments }\end{array}$ \\
\hline Performance & $\begin{array}{c}\text { Reduced Speed } \\
\text { Idling/Minor Stoppages }\end{array}$ \\
\hline Quality & $\begin{array}{c}\text { Defects/Rework } \\
\text { Yield }\end{array}$ \\
\hline
\end{tabular}

In this equation, availability represents the downtime losses of the production line and is defined as the ratio of actual production time and planned production time:

$$
\text { Availability }=\frac{\text { Actual production time }}{\text { Planned production time }}
$$

Planned production time is the total shift time after subtraction of planned breaks (coffee, lunch, etc.) and planned maintenance breaks. All unexpected breaks including longer coffee breaks and breakdowns reduce the availability.

The second component, performance, is defined as the ratio of actual number of products produced and planned number of products:

$$
\text { Performance }=\frac{\text { Total number produced }}{\text { Planned number produced }}
$$

The third and fourth of the big losses define the speed losses leading to the performance metric taking into account all the minor stoppages and operation in less than ideal conditions.

The final contributor, quality, collects the last two of the big losses that are considered as losses due to defects. Quality is defined simply as the ratio of products accepted after quality inspection to the number of all products produced:

$$
\text { Quality }=\frac{\text { Accepted quality products }}{\text { Total number produced }}
$$

As the equation for the OEE (2) is a multiplication of three percentages it cannot be any higher than its lowest component. This means that a production line having near perfect quality and speed can still have a really low OEE if the availability is low. 
OEE can be used as an effective tool for improving the production efficiency but staring at the OEE and its components as such will still give very little information about the possible bottle necks in the production. The reason for data collection should not be acquiring nice graphs [3]. It is also important to note that the OEE is only a measure of internal efficiency and cannot be directly compared to other production plants or lines and there is also some variation in the definition of the OEE and how it is measured [4]. Interpretation of production losses and accuracy of the collected data determine how accurately OEE value can be calculated [5].

Correct analysis of the collected data should be used to move towards improvements in the production process and the OEE and its components will tell if the resulting changes made the impact that was looked for. OEE value can be easily misused and misrepresent a bottleneck if not interpreted careful enough [6]. The main focus should still be in finding them to improve the production capacity. Improving an OEE component by a percentage can have major cost differences compared to other components [7]. Furthermore, concentrating on improving one component, for example performance can lead to reduced quality which in turn can result in a net effect worse than where the process was started from. There is also a pitfall when actions are directed to improving the OEE itself which is not necessarily the same as the most effective way to improve the production and the profit it is supposed to make.

\section{SMALL AND MEDIUM-SIZED ENTERPRISES (SMES) AND TECHNOLOGY TRANSFER (TT)}

Many industrial countries have noticed that small and medium-sized enterprises play an important role in the industry. In spite of the success there seems to be problems in technological development or introduction of new technologies often with these companies. The following are some structural weaknesses that can be observed among small and medium-sized companies on the basis of different studies: - There are seldom deliberate steps for the development of technology, but most of all, it only takes place on the customer's requirements, the pressures caused by the competitive situation or in compliance with relevant laws [8]. - The company does not have enough human resources to introduce new technology internally or through a decision by a management company from outside service providers [9], [10]. The lack of technical experts in the company may also prevent the exploitation of new technologies [11] - The weak financial position of the company slows down technological development projects and weakens the willingness to invest in research and development [12]. TT has been seen as one way of solving the above-mentioned problems in SMEs and can also be called innovation tool (Buratti and Penco, 2001). Also, the implementation of the measurement system developed for the wood industry company mentioned in this study utilized the TT. Technology transfer in general meaning is described in the literature as follows [13]: transferring know-how from donor (such as a university, a research center or a company's $R \& D$ department) to recipients (companies which utilize information).

In the innovation activity of business development, universities play an important role and, through TT, scientific and technological information is made available to companies to exploit in their products or to the development of production processes [14].

\section{MEASURING EQUIPMENT FOR AUTOMATED PRODUCTION LINE}

In this study, a typical automated factory wood processing line and measuring its OEE was studied. Such line consists of several machines doing different processing for the parts (e.g. plane, paint, drying oven, etc.). Typically an automated line machinery is already capable of giving some information relevant to the OEE measurement, at minimum the number of parts produced, but the complete measurement needs some additional hardware and software. The objective of this study was to develop a cost effective, easy to use and reconfigurable solution for production lines such as these.

Although some information could be acquired directly from the machinery the measurement unit was designed to be completely independent of the machinery measured. This approach makes the system easily transformable and replicable and it also works as a precaution as there is no need to connect to any existing machinery electrics. Measurement unit inputs can be connected to existing sensors on the production line if necessary or otherwise beneficial because of the location or ease of the installation, but primarily the aim is to add a new sensor that sees the products running in the line.

The basic operation principle of the system is presented in Fig. 1. It can consist of one or several data collection units which all are nearly identical and chained together in daisy chain configuration with the differential RS-485 serial bus. Each unit is collecting the wanted information from the production line sensors and switches and sending it forward to the next unit until all the data reaches the main unit. The main unit is connected to a PC that has free and open source MariaDB database running where all the data is continuously stored.

The electronics in the data collection units were kept as simple as possible. Simple design makes the system easier to understand and one of the objectives was that no engineering degree is necessarily needed to understand its operation. PICAXE [15] microcontrollers that were originally designed for educational purposes were chosen for the brains of the measuring units. These are very simple to use and to program but still more than capable of doing all the necessary operations needed in this project.

An example schematic of one of the units, the main unit, is presented in Fig. 2. PICAXE input/output ports are used to read the input from the sensors in the production line and are optically isolated. The microcon- 


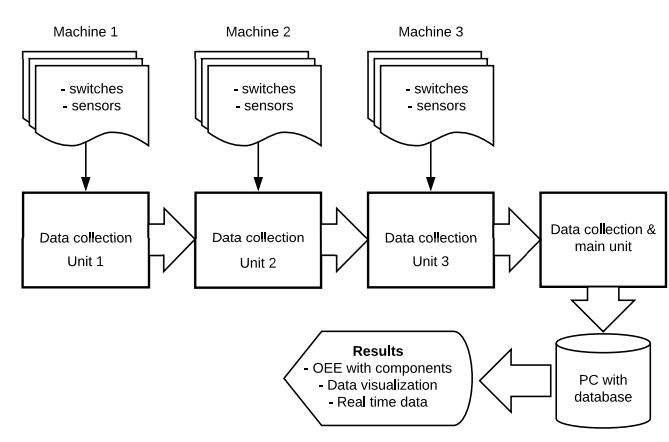

Fig. 1. Data collection principle

troller has hardware support for serial communications and these input/output ports were connected to differential bus transceivers. These transceivers with the help of shielded cabling result in more reliable communications between the measuring units which is a necessity in a noisy production line environment where all types of electrical noise are present.

\section{Measurements}

Typical daily number of pieces going through the line measured in this study was around 3000 and the line was running in two shifts eight hours each and five days a week. The main objective at the beginning was to follow the OEE variation on a weekly basis and to collect data of the stoppages.

The production line consists of four machines each doing different wood processing tasks, drilling, planing, etc. All these machines had mechanical sensors already installed on both inputs and outputs that were used by the production line controlling system to control the flow of processed pieces. These switches had spare contacts that were wired to our measuring system inputs. Each machine has a service key switch which is used by the operators when there is a maintenance break or repairs carried out inside the safety zone of the machine. As these key switches are always and only used when the production is not running and something is done to the machine these were perfect for the measurement system. Service switches had also spare contacts that were wired to the measurement unit inputs. Measurement units were programmed so that after the service key was turned a flashing light was lit to give the operator a sign to choose the reason for the current stoppage using the buttons in the measurement unit.

With the above described operations, the performance of the production could be measured from the input and output switches and the availability could be measured from the service switches to calculate the OEE. The quality component was measured with a separate switch button connected to the main unit. Production line is operated so that all the reproduced parts are run together at the end or beginning of each shift if necessary. These reproduced parts are recognized by the measurement system when the user turns a select switch for the time the reproduction is running.

Availability was calculated from the total shift length, breaks, planned maintenance stops (identified by operators with a defined button after using the service key switch) and serviced time as follows:

$$
\begin{aligned}
& \text { Availability }= \\
& \frac{\text { Shift length - Breaks - Planned stops - Services }}{\text { Shift length - Breaks - Planned stops }}
\end{aligned}
$$

Shift lengths and break lengths were the same for every day, so they were stored as separate settings to the database. Lengths of the planned stops were measured from the service key switch as well as all other stoppages.

An ideal takt time for the production line was measured and decided empirically at the production line output. This value was used to calculate the performance of the line. Production time (PT) relevant for the performance measurement is calculated as follows:

$$
P T=\text { Shift length - Breaks - Planned stops - Services }
$$

After the subtractions from the shift length the significance of human interference to the performance is close to negligible as the fully automated line is always working at its designed speed. In any case, the performance was calculated as follows using the PT value from equation 7 :

$$
\text { Performance }=\frac{\text { Ideal Takt Time } * \text { Parts produced }}{\text { PT }}
$$

Finally, the quality was calculated from the produced and reproduced parts as follows:

$$
\text { Quality }=\frac{\text { Parts produced }- \text { Reproduced parts }}{\text { Parts produced }}
$$

Software was developed for Microsoft Windows operating system to calculate and view the collected data. Overall Equipment Efficiency was calculated from the equations above and the software was developed so that the value can be calculated for any chosen days. The main window of the used software is presented in Fig. 3. Values for OEE and its components are calculated automatically for the user picked date range. Produced pieces together with reproduced items and service times are graphed for the selected time range. One of the most interesting things to follow are the stoppages and more detailed information of those can be viewed with click of a button.

\section{System COSTS}

To verify the cost-effectiveness of the developed measurement system, the total cost of the needed hardware was calculated, and the results are presented in Table II. As the table shows, the system costs were just below 200 euros the biggest expenditures being the microcontrollers and 


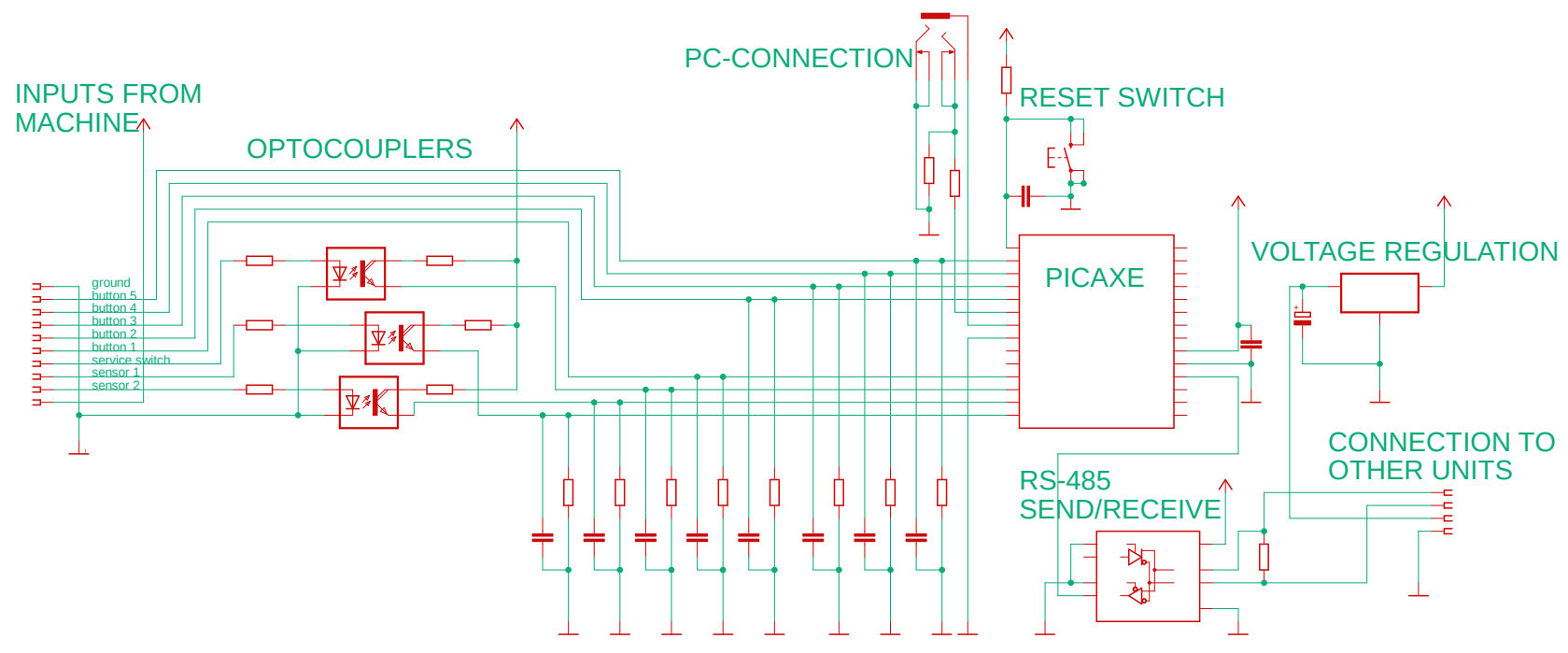

Fig. 2. Schematic of the measuring unit electronics.

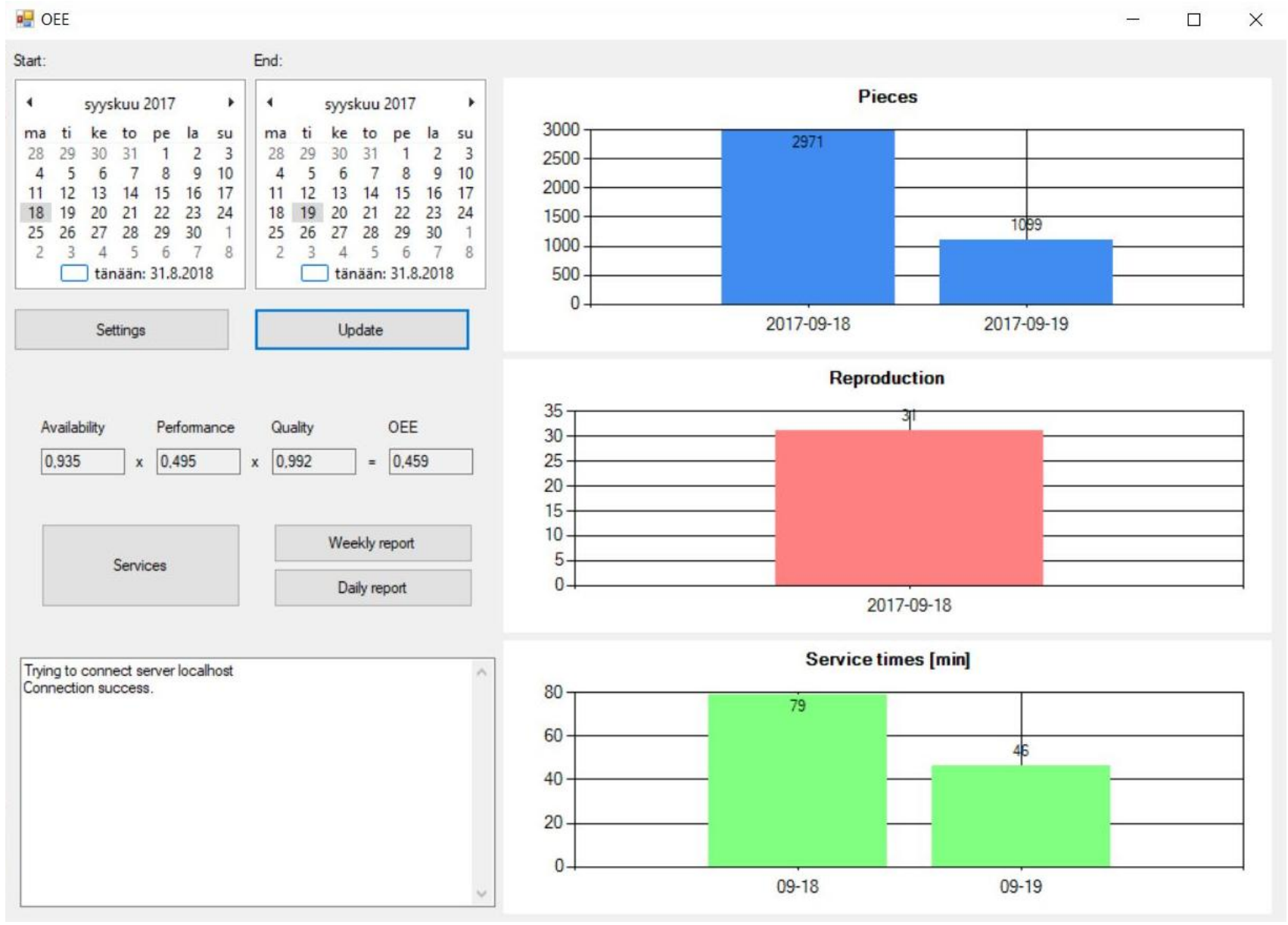

Fig. 3. Main window of the developed OEE software. 
TABLE II

TOTAL SYSTEM COST SEPARATED

\begin{tabular}{l|c}
\hline item & cost [EUR] \\
\hline \hline Microcontrollers & 40 \\
\hline PCBs & 20 \\
\hline Optocouplers & 8,4 \\
\hline Plastic boxes & 48 \\
\hline Resistors, capacitors, connectors & 4 \\
\hline Regulators & 18 \\
\hline Cabling & 15 \\
\hline Buttons and switches & 20 \\
\hline Differential tranceivers & 5 \\
\hline Power supply & 15 \\
\hline \hline total & 193,4 EUR \\
\hline
\end{tabular}

the casings for the electronics. Cabling between the units was carried out with a double shielded four wire cable, two wires used for the power and two for the differential transmission between the units. The completed system left very little room for reducing the costs without making the system more complex. Notably cheaper microcontrollers are on the market, for less than a dollar per unit, but they require a much steeper learning curve and a lot more time to achieve the same end result.

One of the main focuses of this study was to demonstrate how simple hobbyist electronics can be suitable for serious industrial applications. The system was used on a real manufacturing line with all the harsh environmental challenges including electrical interference from high powered processing line and the high amount of dust resulting from the processing. After a timespan of near three years there were no problems with the electronics.

\section{CONCLUSIONS}

Simple and cost-effective system for measuring Overall Equipment Efficiency was presented in this paper. The complete system cost less than 200 euros and was fully functional over the three-year period it was tested on a factory environment. Study shows that there is not necessarily always need for expensive and specialized equipment in the industry applications. Low cost system such as this can attract more SMEs to develop their production.

\section{ACKNOWLEDGMENT}

The authors would like to acknowledge the financial support received from the Council of Oulu Region and the European Union (European Regional Development Fund) for the "KuTuA" project.

\section{REFERENCES}

[1] S. Nakajima, Introduction to TPM. Productivity Press, Cambridge, MA, 1988.

[2] R. Singh, D. Shah, A. Gohil, and M. H. Shahc, "Overall equipment effectiveness (oee) calculation - automation through hardware \& software development," in Procedia Engineering, vol. 51, 2013, pp. 579-584.
[3] K. Ishikawa, Guide to Quality Control. Asian Productivity Organization, Tokyo, 1982.

[4] P. Jonsson and M. Lesshammar, "Evaluation and improvement of manufacturing performance measurement systems - the role of oee," International Journal of Operations \& Production Management, vol. 21, no. 11, pp. 1404-1416, 2001.

[5] R. Hedman, M. Subramaniyan, and P. Almström, "Analysis of critical factors for automatic measurement of oee," in Procedia CIRP, vol. 57, 12 2016, pp. 128-133.

[6] S. E. G. da Costa and E. P. de Lima, "Uses and misuses of the 'overall equipment effectiveness' for production management," in IEEE International Engineering Management Conference, vol. 2, 2002, pp. 816-820 vol.2.

[7] J. Konopka and W. Trybula, "Overall equipment effectiveness (oee) and cost measurement [semiconductor manufacturing]," in Nineteenth IEEE/CPMT International Electronics Manufacturing Technology Symposium, Oct 1996, pp. 137-140.

[8] N. Buratti and L. Penco, "Assisted technology transfer to smes Lessons from an exemplary case," in Technovation 21, 2001, pp. 35-43.

[9] M. Raffa and G. Zollo, Il rapporto fra innovazione tecnologica e organizzazione nelle piccole imprese innovative. Piccola impresa Small Business 2, 1992.

[10] - Economia del software. Edizioni Scientifiche Italiane, Napoli, 1998.

[11] R. Rothwell, The changing nature of the innovation process: implications for SMEs. Oakey, R. (Ed.), New Technology Based Firms in the 1990s. Paul Chapman Publishing, London, 1994

[12] D. Archibugi, R. Evangelista, G. Perani, and F. Rapiti, L'innovazione nelle imprese Italiane: un'analisi dai risultati dell'indagine Istat. Economia e politica industriale 89.

[13] E. Kim, Multinational: preparation for international technology transfer. Gibson, D.V., Williams, F. (Eds.), Technology Transfer: A Communication Perspective. Sage, London, 1990.

[14] M. Ungureanu, N. Pop, and N. Ungureanu, "Innovation and technology transfer for business development." Procedia Engineering 149, 2016, pp. 495-500.

[15] [Online]. Available: http://www.picaxe.com/What-Is-PICAXE 